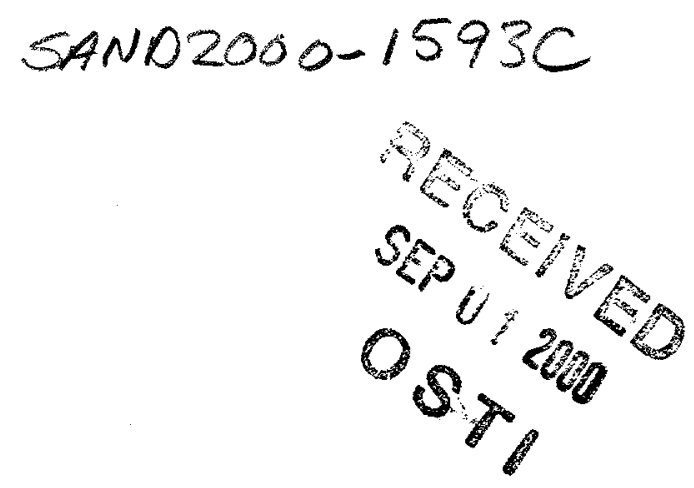

\title{
HIGH AMPLITUDE SECONDARY MASS DRIVE
}

\author{
Christopher W. Dyck, James J. Allen, Robert J. Huber, and Jeffry J. Sniegowski \\ Intelligent Micromachine Department \\ Sandia National Laboratories \\ MS 1080, PO Box 5800 \\ Albuquerque, NM 87185-1080 \\ http://www.mems.sandia.gov
}

\begin{abstract}
In this paper we describe a high amplitude electrostatic drive for surface micromachined mechanical oscillators that may be suitable for vibratory gyroscopes. It is an advanced design of a previously reported dual mass oscillator (Dyck, et. al., 1999). The structure is a 2 degree-of-freedom, parallelplate driven motion amplifier, termed the secondary mass drive oscillator (SMD oscillator). During each cycle the device contacts the drive plates, generating large electrostatic forces. Peak-to-peak amplitudes of $54 \mu \mathrm{m}$ have been obtained by operating the structure in air with an applied voltage of $11 \mathrm{~V}$. We describe the structure, present the analysis and design equations, and show recent results that have been obtained, including frequency response data, power dissipation, and outof-plane motion.
\end{abstract}

\section{INTRODUCTION}

Surface micromachining offers structural complexity, cost advantage, and the potential for a high degree of integration ( $R$. S. Muller, 1990, J. H. Smith, et. al., 1995). Unfortunately, it very difficult to generate large vibration amplitudes with voltages that are compatible with drive and sense electronics. Researches have resorted to using low pressure, hermetically sealed packages to increase the system quality factor. This is practical solution for navigation-grade gyroscopes (degrees/hour), however low pressure packaging introduces reliability concerns due to shock (J. A. Geen, 1998). In applications where degrees/second resolution is required, a large amplitude electrostatic resonator capable of operating at atmospheric pressure is advantageous in gyroscope cost and reliability.
Electrostatic drives usually are either linear comb drives or parallel plate drives. The comb drive can generate high amplitude linear motion but relatively low force. The parallel plate drive generally produces a significantly higher force but with deflections considerably less than the plate separation. Efforts to improve the parallel plate drive, such as motion amplification (Hung, et. al., 1998) or charge control electronics (Seeger, et. al., 1999) have been described. Here, we demonstrate a design using a parallel-plate drive that can produce oscillatory mechanical displacements far in excess of the parallel plate separation.

\section{DEVICE DESCRIPTION}

A SEM photograph and a schematic view of the SMD oscillator are shown in Fig. 1. The structure is composed of two main parts, the primary mass and the secondary mass. The primary mass is anchored to the substrate by four straight beams (primary springs) and has multiple windows etched into it, each encompassing a fixed electrode pair. The fixed electrode pairs are connected in parallel by a thin conductive layer and are termed the right and left electrodes. The structure is electrostatically actuated by applying differential voltage signals of $V_{R}(t)=V_{o}+V_{A C}(t)$ and $V_{L}(t)=V_{o}-v_{A C}(t)$ to the right and left electrodes, respectively. The primary mass motion is limited by the parallel-plate electrode gap. The primary mass area, and thus the area of the entire structure, is approximately $1 \mathrm{~mm}^{2}$.

The secondary mass is suspended within the frame of the primary mass by 4 folded flexures at each of its corners (secondary springs). The plate size is $200 \mu \mathrm{m} \mathrm{x} 450 \mu \mathrm{m}$ and its motion is limited to $\pm 29 \mu \mathrm{m}$ by the inside edge of the primary mass frame. 


\section{DISCLAIMER}

This report was prepared as an account of work sponsored by an agency of the United States Government. Neither the United States Government nor any agency thereof, nor any of their employees, make any warranty, express or implied, or assumes any legal liability or responsibility for the accuracy, completeness, or usefulness of any information, apparatus, product, or process disclosed, or represents that its use would not infringe privately owned rights. Reference herein to any specific commercial product, process, or service by trade name, trademark, manufacturer, or otherwise does not necessarily constitute or imply its endorsement, recommendation, or favoring by the United States Government or any agency thereof. The views and opinions of authors expressed herein do not necessarily state or reflect those of the United States Government or any agency thereof. 


\section{DISCLAIMER}

Portions of this document may be illegible in electronic image products. Images are produced from the best available original document. 


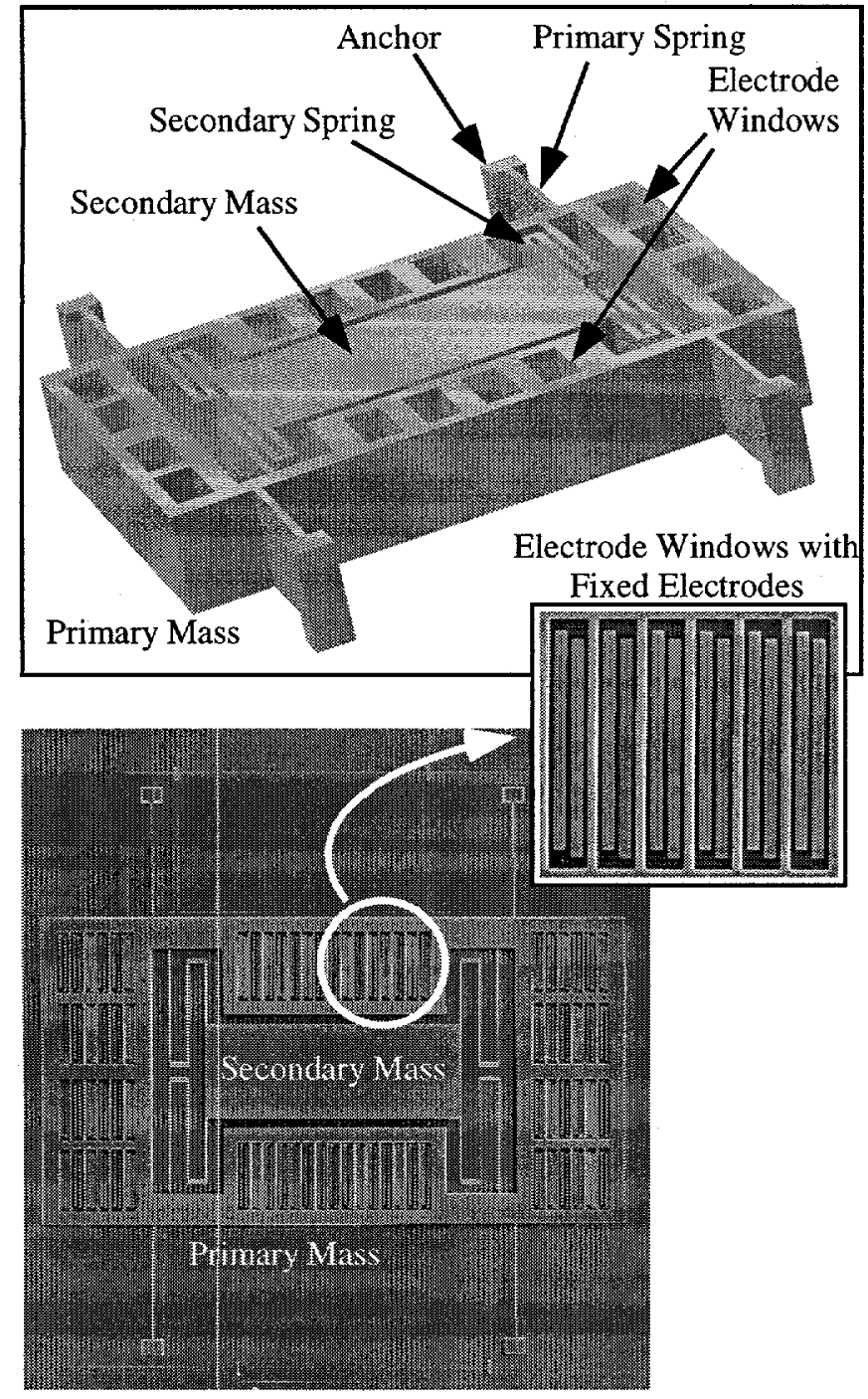

Figure 1: SEM and Schematic of the SMD oscillator.

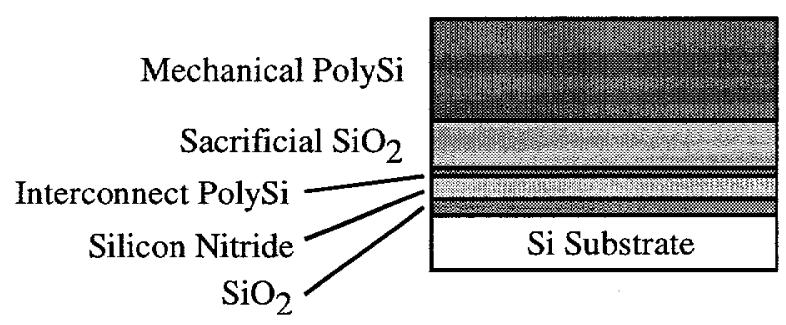

Figure 2: A cross-section of the layers used in the polySi surface micromachining process.

The device was fabricated using a two-level polycrystalline Silicon (polySi) surface micromachining process. A $0.3 \mu \mathrm{m}$ ground plane polySi layer was deposited over a dielectric film stack of Silicon Nitride and Silicon Dioxide of thickness $0.8 \mu \mathrm{m}$ and $0.63 \mu \mathrm{m}$, respectively. The mechanical polySi layer was
$6.65 \mu \mathrm{m}$, and was deposited on a $2 \mu \mathrm{m}$ Silicon Dioxide sacrificial layer. A cross-section of the layers is shown in Fig. 2.

\section{DEVICE ANALYSIS}

The analysis in this work considers the energy transfer between the primary mass and the secondary mass. First, waveform shapes are assumed for the motions of the primary mass and secondary mass that are based on the maximum energy transfer between the two masses. Then, the secondary mass motion is found by calculating the energy transfer from the primary mass to the secondary mass and equating that to the energy dissipated by the secondary mass. Once the secondary mass displacement has been found, the parameters that are necessary to form the assumed waveforms of the primary mass motion and the secondary mass motion are calculated.

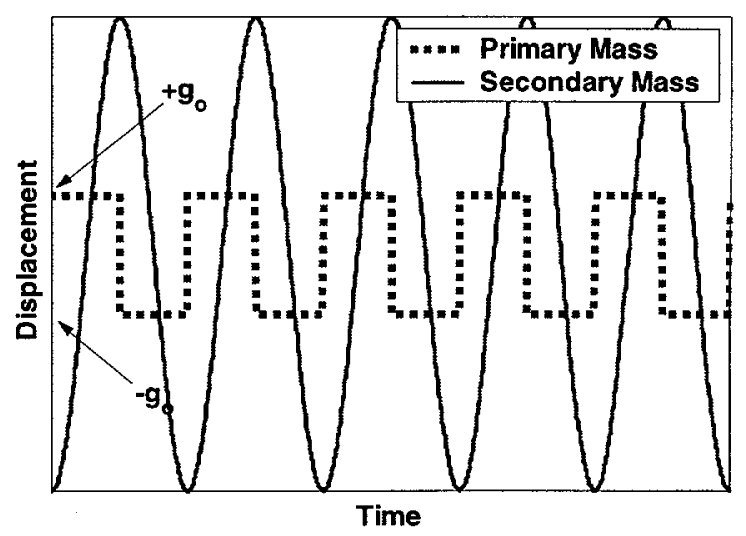

Figure 3: Motion waveforms for the SMD oscillator. The plot shows maximum energy transfer between the primary mass and the secondary mass.

The primary mass is allowed to move the entire length of the gap, $2 \mathrm{~g}_{0}$, during each half cycle, and contact the right and left electrodes. Therefore it is reasonable to assume that the primary mass motion can be approximated with a square wave. This is plotted in Fig. 3. The secondary mass moves within the frame of the primary mass, and its motion will be approximated by a sinusoid. This is justified because of the filtering characteristic of the system steady-state response, and will be discussed further in the next section. This is also plotted in Fig. 3. The waveforms are plotted for the case of maximum energy transfer between the primary mass and the secondary mass. Maximum energy transfer occurs because the primary mass effectively makes an instantaneously transition from one electrode to the other as the secondary mass is fully extended. This happens twice during each cycle.

Since the motion of the primary mass is known, the SMD oscillator can be represented by Fig. 4, where the secondary system is connected to a moving support. The secondary system is represented by $m_{2}, k_{2}$, and $c_{2}$, the mass, the spring 
stiffness, and the viscous damping coefficients, respectively. The position of the secondary mass is $\mathrm{x}_{2}$ and the position of the platform, representing the primary mass, is $x_{1}$.

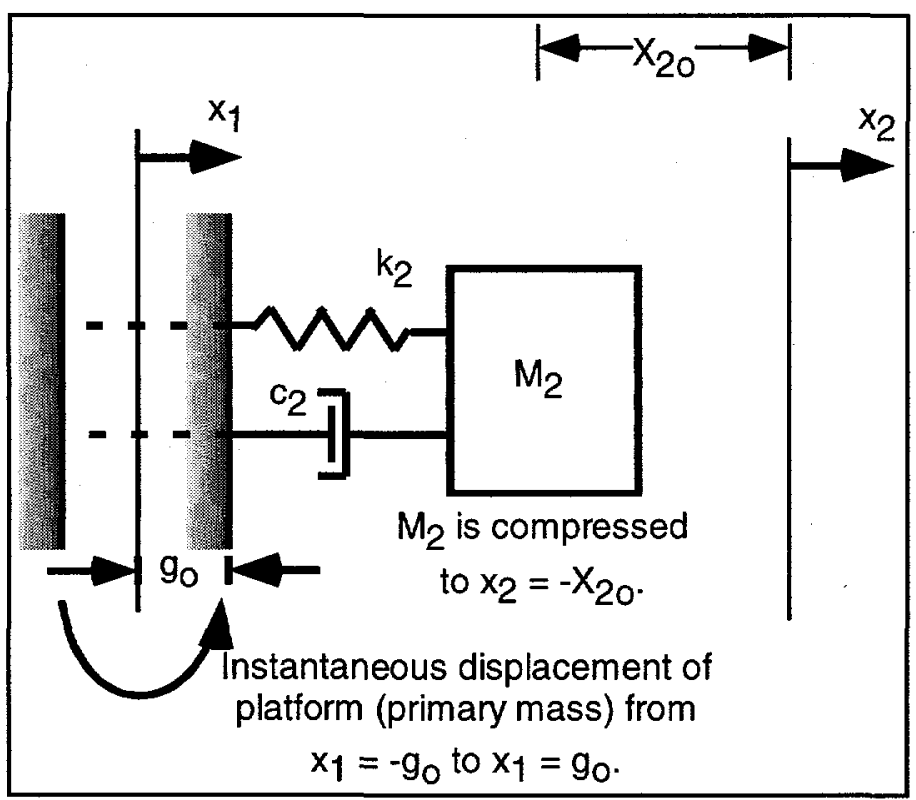

Figure 4: Diagram of the SMD oscillator. The platform (primary mass) moves instantaneously from one electrode to the other while the secondary mass is compressed by its maximum value during one cycle.

The energy dissipated by $m_{2}$ over one cycle is

$$
W=(\pi \omega) c_{2} X_{o 2}^{2}
$$

for a secondary mass steady-state motion of $\mathrm{X}_{\mathrm{o} 2} \cos (\omega \mathrm{t})$.

The energy transfer from the primary mass to the secondary mass is due to the compression and extension of $k_{2}$. When $m_{2}$ is at its maximum amplitude, the primary mass displaces by $2 \mathrm{~g}_{0}$ and injects energy into the system. This is shown in Fig. 4. The energy injected into the system is the difference in spring energy of $k_{2}$ before and after $m_{1}$ moves,

$$
W_{k}=2 \cdot\left[\frac{1}{2} k_{2}\left(X_{2 o}+g_{o}\right)^{2}-\frac{1}{2} k_{2}\left(X_{2 o}-g_{o}\right)^{2}\right],
$$

where $g_{o}$ is the parallel-plate electrode gap. The factor of 2 in front of the brackets is needed because the platform makes two transitions during a single cycle. Eq. (2) simplifies further to

$$
W_{k}=4 k_{2} g_{o} X_{2 o} \text {. }
$$

The secondary mass amplitude is found by equating (1) and (3) and solving for $\mathrm{X}_{20}$,

$$
X_{2 o}=\frac{4 k_{2} g_{a}}{\pi \omega c_{2}} .
$$

Reasonable parameters in a surface micromachining process are $\mathrm{k}_{2}=1 \mathrm{~N} / \mathrm{m}, \mathrm{g}_{\mathrm{o}}=1 \mu \mathrm{m}, \omega=2 \pi(10 \mathrm{kHz})$, and $\mathrm{c}_{2}=1 \mathrm{~N} /(\mathrm{m} / \mathrm{s})$, resulting in $\mathrm{X}_{02}=20.3 \mu \mathrm{m}$.

The second half of the design involves calculating $\mathrm{k}_{1}$ and the drive voltage that are necessary to place the structure in the contact mode. This is summarized in Fig. 5. The secondary mass is maximally compressed, as before, and the primary mass is assumed to be contacting the left electrode before the transition. A voltage, $V_{R}(t)$ is applied to the right electrode, and the left electrode and $m_{1}$ are set to ground. Initially, $V_{R}(t)=0$ volts, and the left electrode has a positive voltage. At $t=0$, the transition time, $V_{R}(t)$ becomes positive, and the left electrode becomes ground. $m_{1}$ is also set to ground. The stated problem is then to calculate what $V_{R}$ and $k_{1}$ are necessary such that $m_{1}$ will clamp to the right electrode from its initial position at the left electrode. The geometry, $\mathrm{X}_{20}, \mathrm{k}_{2}$, and $\omega$, are assumed to be known from the analysis in the previous paragraphs.

Two cases are considered in the analysis. The first case is the quasi-static analysis. The voltage on the right electrode is calculated that makes the primary mass unstable at any position in the gap. This way the primary mass will always clamp to the right electrode, from its initial position at the left electrode. This analysis neglects the kinetic energy of $m_{1}$ that it acquires as it transitions the gap from the left to the right electrode. The second case is the dynamic analysis. The amount of energy acquired by the primary mass during the transition is included in this analysis. Energy losses from damping are neglected in the dynamic analysis, resulting in a calculated drive voltage level that represents a lower limit on the required contact mode drive voltage.

Quasi-static Analysis: The forces acting on $\mathrm{m}_{1}$ are shown in Fig. 5. $F_{e}$ is the electrostatic force given by

$$
F_{e}=\frac{C_{o}}{2 g_{o}}\left[\left(\frac{V_{R}}{1-\frac{x_{1}}{g_{o}}}\right)^{2}-\left(\frac{V_{L}}{1+\frac{x_{1} / g_{o}}{g_{o}}}\right)^{2}\right],
$$

and $F_{1}$ and $F_{2}$ are the spring forces. $V_{R}$ and $V_{L}$ are square waves that are $180^{\circ}$ out of phase and toggle between 0 Volts and $V_{o}$ Volts. When $V_{R}=V_{o}$ and $V_{L}=0, F_{e}$ reduces to

$$
F_{e}=\frac{C_{o}}{2 g_{o}}\left(\frac{V_{R}}{1-x_{1} / g_{o}}\right)^{2} .
$$




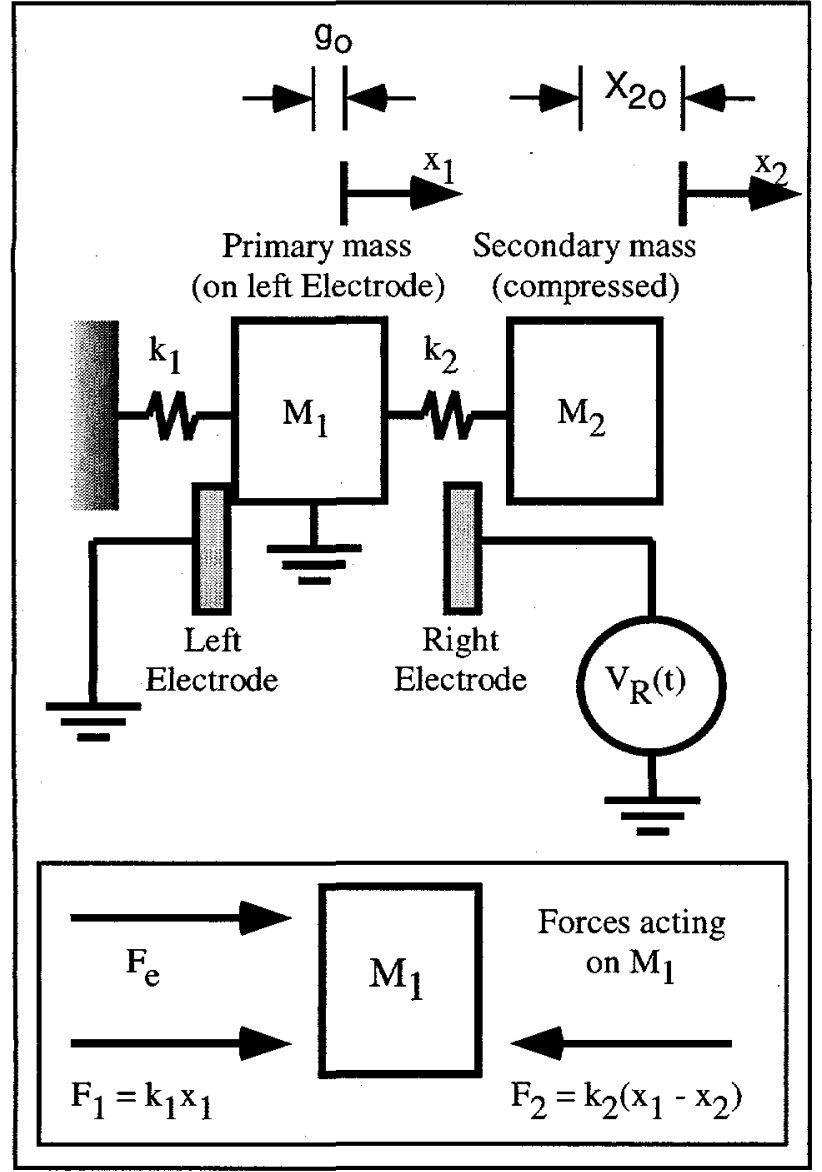

Figure 5: SMD oscillator diagram that is used to find the voltage and $k_{1}$ values required for the contact mode.

When the electrostatic and spring forces are summed on the primary mass, the following equation results,

$$
\frac{C_{o}}{2 g_{o}}\left(\frac{V_{R}}{1-x_{1} / g_{o}}\right)^{2}=k_{1} x_{1}-k_{2}\left(x_{1}-x_{2}\right) \text {. }
$$

Solving for the voltage on the right electrode results in

$$
V_{R}^{2}=\frac{2 g_{o}}{C_{o}}\left(1-\frac{x_{1}}{g_{o}}\right)^{2}\left[k_{1} x_{1}+k_{2}\left(x_{1}-x_{2}\right)\right] .
$$

To find the voltage on the right electrode that makes the primary mass unstable at any position within the gap, a method analogous to what Nathanson, et. al. used is applied here. They set $\mathrm{dV} / \mathrm{dx}=0$ for a pair of parallel-plate electrodes biased with voltage $\mathrm{V}$. This can be interpreted as finding position in the gap where a zero change in the applied voltage results in a finite displacement in $x$. This is the criterion for instability.
Proceeding with this method, the derivative of Eq. (8) is calculated and set to zero, resulting in the following root equation in $x_{1}$ :

$$
x_{1}^{2}+\left[-\frac{2 k_{2} x_{2}}{3\left(k_{1}+k_{2}\right)}-4 \frac{g_{o}}{3}\right] x_{1}+g_{o}\left[\frac{g_{o}}{3}+\frac{2 k_{2} x_{2}}{3\left(k_{1}+k_{2}\right)}\right]=0 .
$$

This is further reduced to

$$
\left(x_{1}-g_{o}\right)\left[x_{1}-\left(\frac{g_{o}}{3}+\frac{2 k_{2} x_{2}}{3\left(k_{1}+k_{2}\right)}\right)\right]=0 \text {. }
$$

The solution to Eq. (11) is the pull-in length,

$$
x_{1}=X_{1 P I}=\frac{g_{o}}{3}+\frac{2 k_{2} x_{2}}{3\left(k_{1}+k_{2}\right)} \text {. }
$$

For the situation in Fig. 5, $x_{2}=-\mathrm{X}_{20}$, and Eq. (12) reduces to

$$
X_{1 P I}=\frac{g_{o}}{3}-\frac{2 k_{2} X_{2 o}}{3\left(k_{1}+k_{2}\right)} \text {. }
$$

When $\mathrm{X}_{20}=0$, Eq. (12) reduces to the familiar result of $\mathrm{g}_{\mathrm{o}} / 3$, the pull-in length of a parallel-plate electrode pair described by Nathanson, et. al. The effect of compressing $m_{2}$ is to decrease the position of stability, moving it further toward the left electrode. Eq. (12) is substituted into Eq. (8) to find the pull-in voltage

$$
V_{R}=V_{1 P I}=\left\{\begin{array}{r}
\frac{4\left(k_{1}+k_{2}\right)}{C_{o}}\left[\left(\frac{g_{o}}{3}\right)^{2}-\left(\frac{2 k_{2} X_{2 o}}{3\left(k_{1}+k_{2}\right)}\right)^{2}\right] \\
-\frac{4 k_{2} X_{2 o}}{C_{o}}\left[\frac{g_{o}}{3}-\frac{2 k_{2} X_{2 o}}{3\left(k_{1}+k_{2}\right)}\right]
\end{array}\right\}^{\frac{1}{2}} .
$$

$\mathrm{X}_{1 \mathrm{PI}}$ and $\mathrm{V}_{1 \mathrm{PI}}$ are plotted versus $\mathrm{k}_{1}$ for $\mathrm{C}_{\mathrm{o}}=250 \mathrm{ff}\left(\mathrm{g}_{\mathrm{o}}=1 \mu \mathrm{m}\right)$ in Figure 6. The parameters, $\mathrm{k}_{2}=1 \mathrm{~N} / \mathrm{m}, \omega=2 \pi(10 \mathrm{kHz})$, and $\mathrm{X}_{\mathrm{o} 2}$ $=20.3 \mu \mathrm{m}$ are used again. For $\mathrm{k}_{1}=15.8 \mathrm{~N} / \mathrm{m}$, the pull-in length is $\mathrm{X}_{1 \mathrm{PI}}=-0.473 \mu \mathrm{m}$ and the pull-in voltage is $\mathrm{V}_{1 \mathrm{PI}}=14.64$ Volt. The effect of increasing the primary spring, $\mathrm{k}_{1}$ is to increase the pull-in length toward $\mathrm{g}_{0} / 3$ and to decrease the pull-in voltage.

Dynamic Analysis: Eq. (13) overestimates the voltage required to place the device in the contact mode. When $m_{1}$ is initially clamped to the left electrode and released, it will gain kinetic energy, which is ignored in the quasi-static analysis. 

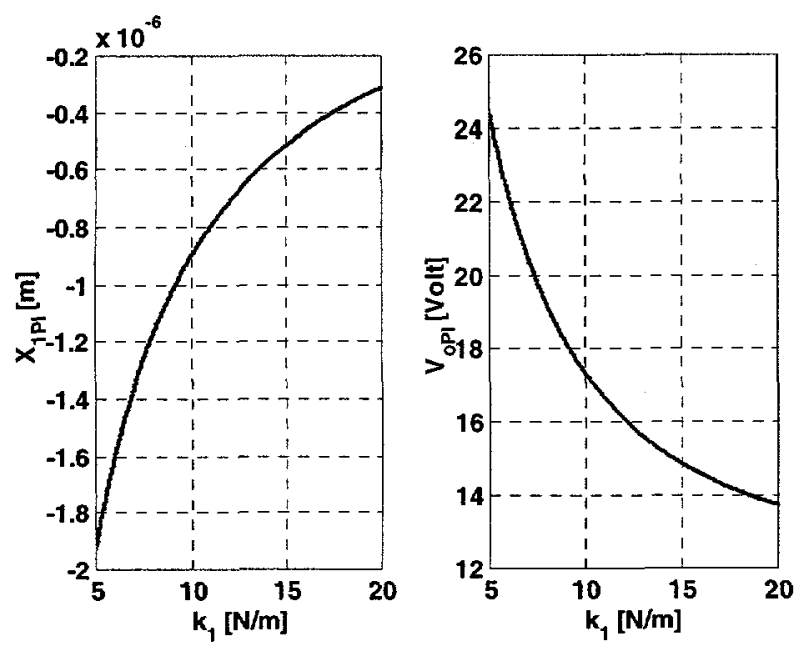

Figure 6: Plots of the pull-in length and the pull-in voltage vs. $k_{1}$ when the structure is in the contact mode.

To account for kinetic energy, consider Fig. 7 where the left and right sides of Eq. (7) have been plotted. These are the electrostatic and spring forces acting on $\mathrm{m}_{1}$. The positions, $\mathrm{x}_{1} / \mathrm{g}_{\mathrm{o}}=\mathrm{X}_{\mathrm{eq}}$ and $\mathrm{x}_{\mathrm{l}} / \mathrm{g}_{\mathrm{o}}=\mathrm{X}_{\text {neq }}$ in Fig. 7 are the equilibrium and nonequilibrium intersection points of the force curves. In the quasi-static case previously described, $\mathrm{m}_{1}$ would have come to rest at $X_{e q}$ if the drive voltage, $V_{R}$ were not greater than $V_{1 P I}$. The mechanical and electrical potential energies are the areas under their respective force curves. In the dynamic case, when the primary mass is released from the left electrode, it moves toward $X=X_{e q}$, the static equilibrium point. From $x=-g_{o}$ to $x$ $=\mathrm{X}_{\mathrm{eq}}, \mathrm{m}_{1}$ obtains kinetic energy equal to the difference in areas under the force curves, denoted as $A_{1}$ in Fig. 7. If this kinetic energy exceeds the potential energy barrier, denoted as $A_{2}$ in Fig. 7 , the primary mass will continue moving to the right electrode and clamp. If it is less than $A_{2}$, the mass will not clamp and will settle at the equilibrium point, $\mathrm{X}_{\mathrm{eq}}$.

$V_{1 P I}$ and $k_{1}$ are solved using two simultaneous equations. The first finds the non-equilibrium points, $X_{\text {neq }}$, for varying $V_{1 P I}$ and $k_{1}$ values. The second looks for $A_{1} \geq A_{2}$ (the electrostatic energy, $W_{e}$ exceeds the spring energy $W_{k}$ ) using the $X_{\text {neq }}$ values from the first equation.

The non-equilibrium points, $X_{\text {neq }}$ are found by equating the electrostatic and the spring forces, which was done in Eq. (7). This is expanded to the following root equation:

$$
\begin{gathered}
x_{1}^{3}+x_{1}^{2}\left(-2 g_{o}-\frac{k_{2} x_{2}}{k_{1}+k_{2}}\right)+x_{1}\left(g_{o}{ }^{2}+\frac{2 g_{o} k_{2} x_{2}}{k_{1}+k_{2}}\right) \\
+\frac{g_{o}{ }^{2}}{k_{1}+k_{2}}\left(-k_{2} x_{2}-\frac{C_{o} V_{R}{ }^{2}}{2 g_{o}}\right)=0
\end{gathered}
$$

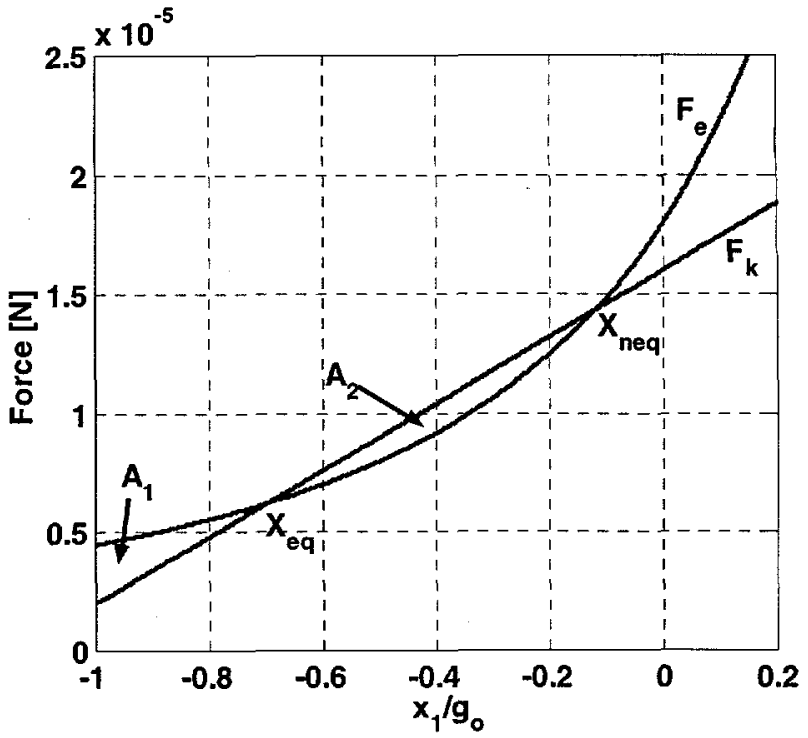

Figure 7: Plots of the electrostatic and spring forces, $F_{\mathrm{e}}$ and $F_{k}$. The difference, $A_{1}-A_{2}$ is the kinetic energy possessed by $m_{1}$ at $x=X_{\text {neq. }}$

$$
\begin{array}{r}
x_{1}^{3}+x_{1}{ }^{2}\left(-2-\frac{k_{2} x_{2}}{k_{1}+k_{2}}\right)+x_{1}\left(1+\frac{2 k_{2} x_{2}}{k_{1}+k_{2}}\right) \\
+\left(-\frac{k_{2} x_{2}}{k_{1}+k_{2}}-\frac{C_{o} V_{R}^{2}}{2 g_{o}{ }^{2}\left(k_{1}+k_{2}\right)}\right)=0
\end{array}
$$

when the primary mass motion, $\mathrm{x}_{1}$ is normalized to the gap, $\mathrm{g}_{0}$. The greatest real root over the interval of $x_{1}=(-1,1)$ is $X_{\text {neq. }}$. This value is best found by using software.

The electrostatic energy is the integral of Eq. (6)

$$
W_{e}=\frac{C_{o} V_{R}{ }^{2}}{2 g_{o}} \int_{0}^{X_{\text {neq }}} \frac{d x_{1}}{\left(1-x_{1} / g_{o}\right)^{2}} .
$$

Normalizing $\mathrm{x}_{1}$ to $\mathrm{g}_{\mathrm{o}}$ and evaluating this expression gives

$$
W_{e}=\frac{C_{o} V_{R}^{2}}{2}\left(\frac{1}{1-X_{n e q}}-\frac{1}{2}\right) \text {. }
$$

The spring energy is the integral of the right side of Eq. (7)

$$
W_{k}=\left(k_{1}+k_{2}\right) \int_{-g_{o}}^{X_{\text {neq }}} x_{1} d x_{1}-k_{2} x_{2} \int_{-g_{o}}^{X_{\text {neq }}} d x_{1} \text {. }
$$

Normalizing $\mathrm{x}_{1}$ to $\mathrm{g}_{\mathrm{o}}$, and evaluating the expression results in

and further to 


$$
W_{k}=\frac{g_{o}{ }^{2}\left(k_{1}+k_{2}\right)}{2}\left(X_{n e q}{ }^{2}-1\right)-g_{o}{ }^{2} k_{2} x_{2}\left(X_{n e q}+1\right) .
$$

$W_{e}-W_{k}$ is plotted in Fig. 8 for the set of $X_{\text {neq }}$ values generated in Eq. (15). The parameters, $\mathrm{k}_{2}=1 \mathrm{~N} / \mathrm{m}, \mathrm{g}_{0}=1 \mu \mathrm{m}, \omega=2 \pi(10$ $\mathrm{kHz}$ ), and $X_{02}=20.3 \mu \mathrm{m}$ are again assumed. $V_{R}=12 \mathrm{~V}, 13 \mathrm{~V}$, and $14 \mathrm{~V}$ are used in the plots. The SMD oscillator is in the contact mode for primary spring values that correspond to $\mathrm{W}_{\mathrm{e}}-$ $W_{k} \geq 0$, equivalent to $A_{2} \geq A_{1}$ in Fig. 7. For $k_{1}=15.8 \mathrm{~N} / \mathrm{m}$, the pull-in voltage is $\mathrm{V}_{1 \mathrm{PI}}=14$ Volt, slightly less than the pull-in voltage calculated using the quasi-static analysis.

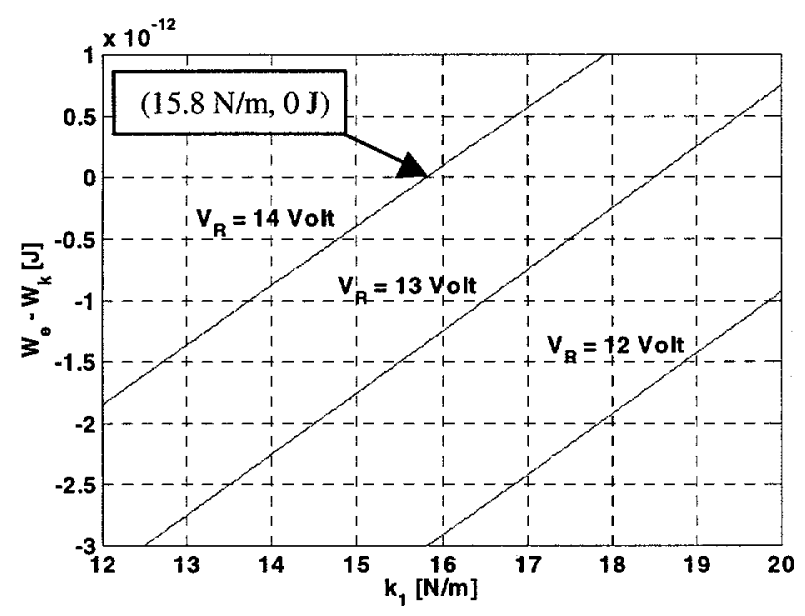

Figure 8: Plots of $W_{e}-W_{k}$ for three drive voltage values. The SMD oscillator is in the contact mode for $k_{1}$ values corresponding to $W_{e}-W_{k} \geq 0$.

\section{FREQUENCY RESPONSE}

The frequency response can be solved for frequencies near $f=f_{A R}$. Over this range, the square wave behavior of the primary mass can be approximated by a sinusoid due to the filtering characteristics of the SMD oscillator. This is convenient for both the design and analysis of the system.

The Fourier series of the primary mass displacement waveform (Fig. 3) is

$$
\begin{aligned}
x_{1}(t)= & \frac{4 g_{o}}{\pi} \cos (\omega t)- \\
& \frac{4 g_{o}}{\pi}\left(\frac{1}{3}\right) \cos (3 \omega t)+ \\
& \frac{4 g_{o}}{\pi}\left(\frac{1}{5}\right) \cos (5 \omega t)-
\end{aligned}
$$

The secondary mass steady-state time response, $x_{2}(t)$ is a superposition of responses,

$$
x_{2}(t)=h(t) *\left(\begin{array}{r}
\frac{4 g_{o}}{\pi} \cos (\omega t)- \\
\frac{4 g_{o}}{\pi}\left(\frac{1}{3}\right) \cos (3 \omega t)+ \\
\frac{4 g_{o}}{\pi}\left(\frac{1}{5}\right) \cos (5 \omega t)-\quad \ldots
\end{array}\right),
$$

where $h(t)$ is the time domain equation of the steady-state system transfer function, $H(j \omega)=X_{2}(j \omega) / X_{1}(j \omega)$, given as

$$
H(j \omega)=\frac{1}{1-\left(\frac{\omega}{\omega_{A R}}\right)^{2}+j \frac{\omega}{\omega_{A R} Q}}
$$

In the frequency domain, Eq. (21) becomes

$$
\begin{aligned}
x_{2}(t)= & \frac{4 g_{o}}{\pi}|H(j \omega)| \cos \left(\omega t+\phi_{1}\right)- \\
& \frac{4 g_{o}}{\pi}\left(\frac{1}{3}\right) H(j 3 \omega) \mid \cos \left(3 \omega t+\phi_{3}\right)+ \\
& \frac{4 g_{o}}{\pi}\left(\frac{1}{5}\right) H(j 5 \omega) \mid \cos \left(5 \omega t+\phi_{5}\right)-
\end{aligned}
$$

where

$$
|H(j n \omega)|=\frac{1}{\sqrt{\left[1-\left(n \omega / \omega_{A R}\right)^{2}\right]^{2}+\left(n \omega / \omega_{A R} Q\right)^{2}}}
$$

and

$$
\phi_{n}=-\tan ^{-1}\left[\frac{n \omega / \omega_{A R}}{1-\left(n \omega / \omega_{A R}\right)^{2}}\right]
$$

are the magnitude and phase of $H(j \omega)$, evaluated at the fundamental frequency and the harmonics. In series form, Eq. (23) is

$$
x_{2}(t)=\frac{4 g_{o}}{\pi} \sum_{n=1,3,5, \ldots}(-1)^{\frac{n-1}{2}}\left(\frac{1}{n}\right)|H(j n \omega)| \cos \left(n \omega t+\phi_{n}\right) .
$$

Eq. (26) is an infinite series response. The higher order, odd harmonic Fourier series coefficients of the primary mass motion decrease by $1 / \mathrm{n}$, where $\mathrm{n}$ is the harmonic order $(1,3,5, \ldots)$. 
The amplitude frequency response, $|\mathrm{H}(j \omega)|=\left|\mathrm{X}_{2}(j \omega) / \mathrm{X}_{1}(j \omega)\right|$ decreases as $\left(\omega / \omega_{A R}\right)^{2}$ for $\omega \gg \omega_{A R}$, effectively behaving as a $2^{\text {nd }}$ order mechanical filter with $40 \mathrm{~dB} /$ decade attenuation. The $3 \mathrm{~dB}$ frequency is found by setting $|\mathrm{H}(\mathrm{j} 0)|=(0.5)^{1 / 2}$, and by assuming a $\mathrm{Q}$ corresponding to a lightly damped system,

$$
\omega_{3 d B} \approx(1+\sqrt{2})^{1 / 2} \omega_{A R}=(1.55) \omega_{A R} .
$$

For harmonics greater than $\omega_{3 d B}$, the product of $|\mathrm{H}(\mathrm{j} \omega)|$ and the Fourier coefficients is negligible in the solution, and Eq. (26) reduces to

$$
x_{2}(t)=|H(j \omega)| \frac{4 g_{o}}{\pi} \cos (\omega t+\phi)
$$

In the frequency domain, this is

$$
\frac{X_{2}(j \omega)}{g_{o}}=H(j \omega) \frac{4}{\pi} .
$$

Equation (29) is the system frequency response, and is valid for frequencies below (1.55) $\omega_{\mathrm{AR}}$.

\section{RESULTS}

The mechanical parameters and the geometry of the SMD oscillator are listed in Table 1. The electrode gap, $g_{0}$, was measured by scanning electron micrograph, and the mechanical polySi layer thickness, $h$, was measured from control wafers by an ellipsometer. The equilibrium capacitance, $\mathrm{C}_{0}$, was calculated from the geometry measurements, and neglects fringe field effects. The primary and secondary masses were calculated assuming a polySi density of $2.33 \mathrm{~g} / \mathrm{cm}^{3}$.

Table 1: Measured mechanical parameters and geometry

\begin{tabular}{|c|c|c|}
\hline \multicolumn{3}{|c|}{ Mechanical Parameters } \\
\hline Primary Spring & $\mathrm{k}_{1}$ & $9.43 \mathrm{~N} / \mathrm{m}$ \\
\hline Primary Mass & $\mathrm{m}_{1}$ & $40.4 \times 10^{-10} \mathrm{~kg}$ \\
\hline Secondary Spring & $\mathrm{k}_{2}$ & $0.874 \mathrm{~N} / \mathrm{m}$ \\
\hline Secondary Mass & $\mathrm{m}_{2}$ & $13.9 \times 10^{-10} \mathrm{~kg}$ \\
\hline Secondary Damping & $\mathrm{c}_{2}$ & $2.90 \times 10^{-6} \mathrm{~N} /(\mathrm{m} / \mathrm{s})$ \\
\hline Antiresonant Frequency & $\mathrm{f}_{\mathrm{AR}}$ & $3990 \mathrm{~Hz}$ \\
\hline Quality Factor & $\mathrm{Q}$ & 12 \\
\hline \multicolumn{3}{|c|}{ Geometry } \\
\hline Mech. PolySi Thickness & $\mathrm{h}$ & $6.65 \mu \mathrm{m}$ \\
\hline Electrode Gap & $\mathrm{g}_{0}$ & $2.4 \mu \mathrm{m}$ \\
\hline Electrode Length & $\mathrm{l}$ & $9.37 \mathrm{~mm}$ \\
\hline Equilibrium Capacitance & $\mathrm{C}_{\mathrm{o}}$ & $230 \mathrm{ff}$ \\
\hline
\end{tabular}

The remaining parameters were measured in the lab with the setup described in an earlier work (Dyck). Briefly, the released wafers were placed on a probe station, and illuminated with a white light strobe source. The displacements of the primary mass and the secondary mass were observed with a calibrated video measurement unit to a resolution of approximately $0.25 \mu \mathrm{m}$. The strobe source was used to create an aliased image of the oscillating structure. Modulating the strobe frequency changed the velocity of the image of the moving structure, allowing detailed amplitude measurements to be recorded.

The antiresonant frequency, $f_{A R}$, was measured by operating the structure in the non-contact mode and recording the frequency where the movement of $m_{1}$ was a minimum. Operation of the structure in the non-contact mode has been described previously (Dyck). Once $\mathrm{f}_{\mathrm{AR}}$ had been calculated, the secondary spring was calculated as $\mathrm{k}_{2}=\mathrm{m}_{2} \omega_{\mathrm{AR}}{ }^{2}$. $\mathrm{Q}$ was calculated as the measured ratio, $\mathrm{X}_{2}\left(\mathrm{j} \omega_{\mathrm{AR}}\right) / \mathrm{X}_{1}\left(\mathrm{j} \omega_{\mathrm{AR}}\right)$.

A dc voltage referenced to the shuttle was applied to one of the electrodes and ramped up slowly until the shuttle clamped at $\mathrm{V}=8.37$ Volt. This value was used to calculate $\mathrm{k}_{1}$ from the equation for the pull-in voltage (Nathanson, 1967),

$$
k_{1}=\frac{27}{8} \frac{C_{o} V_{P I}^{2}}{g_{o}{ }^{2}} .
$$

The amplitude frequency response (AFR) of the SMD oscillator in the contact mode was measured using the test setup described earlier. The structure was placed in the contact with differential sinusoidal signals of offset and amplitude of $V_{0}=$ 5.5 Volt and $\mathrm{V}_{\mathrm{AC}}=5.5$ Volt, respectively. The signal frequency was increased from $3.4 \mathrm{kHz}$ to $4.2 \mathrm{kHz}$ and $\mathrm{X}_{2 \mathrm{o}}$ was recorded. This is plotted in Fig. 9, along with the amplitude of the frequency response of Eq. (29) (using measured values). The structure stays in the contact mode below the transition frequency of $3.8 \mathrm{kHz}$. The transition frequency will be defined as the frequency of maximum amplitude of the structure as it is moving from the contact mode to the non-contact mode. Above the transition frequency the structure falls out of the contact mode. The maximum amplitude obtained by the structure at the transition frequency was $24.35 \mu \mathrm{m}$.

As a preliminary test of the reliability of the SMD oscillator, the structure was allowed to run for an extended period in the contact mode. The structure was left on a probe station for 2.2 billion cycles (159.5 hours). The ambient temperature and humidity in the lab are $27^{\circ} \mathrm{C}$ and $40 \%$, respectively. The structures were not treated with any special antistiction layers before the test. The test was terminated, not because the device failed, but to preserve the wafer for future testing.

The power dissipation of the contact mode was measured by recording the voltage across a $10 \mathrm{k} \Omega$ resistor placed in series with the structure and ground. At all frequencies above $0.5 \mathrm{~Hz}$, the structure showed no dc power dissipation during the portions of the cycle that the shuttle was in contact with either of the parallel-plate electrodes. Fig. 10 shows a typical trace (ac coupling on). The sinusoidal trace is the drive voltage and 
the second trace is the voltage across the resistor. The voltage transients of the second trace appeared during the transition of the shuttle from one electrode to the other. The traces were also observed with the ac coupling turned off. There was no observable change in the trace, indicating the absence of a dc offset voltage across the resistor. Below $0.5 \mathrm{~Hz}$, intermittent and variable dc offset voltages were recorded, corresponding to current readings in the milliamp range.

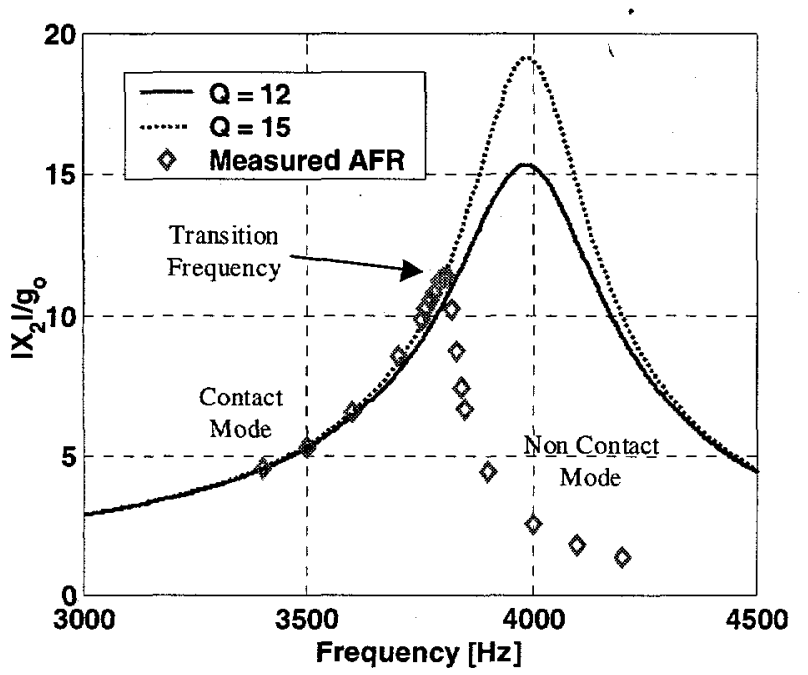

Figure 9: Measured AFR for the SMD oscillator in the contact mode, overlaid with the AFR of Eq. (29). The structure falls out of the contact mode above $3.8 \mathrm{kHz}$.

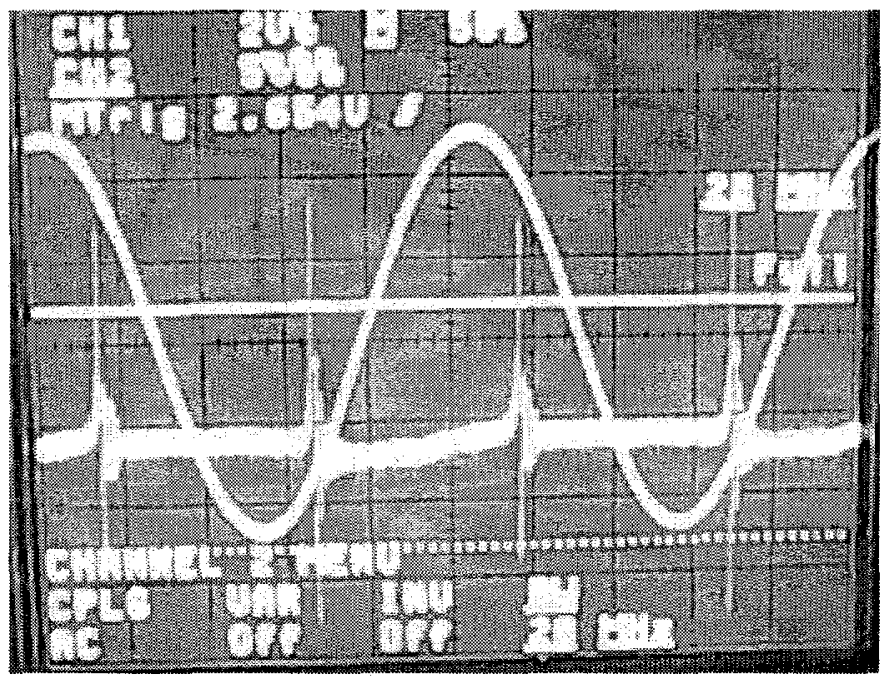

Figure 10: Voltage traces that were used to measure power dissipation. The pulses appeared as the structure moved from one electrode to the other.

The out-of-plane motion of the SMD oscillator was measured using a white light strobe source connected to a microscope with a $550 \mathrm{~nm}$ filter and an interference objective.
By aliasing the image of the moving structure, the motion of the interference fringes was observed. The interference fringes of the primary mass moved by approximately $1 / 2$ of a fringe, equivalent to $225 \mathrm{~nm}$ of out-of-plane motion. The interference fringes of the secondary mass moved less than $1 / 10^{\text {th }}$ of a fringe $(22.5 \mathrm{~nm})$, the resolution of our test setup.

\section{DISCUSSION}

The measured AFR in Fig. 9 shows that the SMD oscillator is capable of large amplitudes at modest drive voltages in open air operation. The secondary mass amplitude at the transition frequency was $24.35 \mu \mathrm{m}$ using an 11 Volt drive signal. Theory further predicts that if the structure could have been driven at $f$ $=\mathrm{f}_{\mathrm{AR}}$, an amplitude of $36.7 \mu \mathrm{m}$ would have been possible.

Although the amplitudes measured here were high, it would not be suitable to operate a vibratory gyroscope at the transition frequency because of stability issues associated with maintaining a constant secondary mass amplitude. The structure could, however, be redesigned with a gap of $1 \mu \mathrm{m}$, as discussed in the example in the theoretical section. High amplitudes at reasonable drive voltages could still be maintained this way.

Equations were derived to predict the secondary mass amplitude at $f=f_{A R}$. These equations assumed maximum energy transfer between the primary mass and the secondary mass, leading to the waveforms shown in Fig. 3. Non-ideal behavior occurs because the primary mass motion is not a square wave. The transition times are finite, and the energy transfer from the primary mass to the secondary mass becomes less efficient. At drive voltages close to $\mathrm{V}_{\mathrm{IPI}}$, the primary mass motion deviates significantly from a square wave, and lower secondary mass motions can be expected. This has been observed in simulations, and this has been observed in the lab at frequencies below the transition frequency. At voltages that are significantly greater than $\mathrm{V}_{1 \mathrm{PI}}$, the primary mass motion behaves more ideally. The primary mass transitions more quickly across the gap because of the higher drive force, and more energy is transferred to the secondary mass.

If it is necessary to have a more accurate prediction of the secondary mass amplitude, the primary mass waveform needs to be accurately recorded. Once this has been done, the $1^{\text {st }}$ Fourier series coefficient of the primary mass waveform can be calculated and substituted into Eq. (29). The result is a more accurate AFR near $\mathrm{f}=\mathrm{f}_{\mathrm{AR}}$.

Equations were also derived to predict the conditions necessary to drive the structure in the contact mode. The results of the quasi-static analysis and the dynamic analysis were similar. It is reasonable then to use the more user-friendly equations in the quasi-static analysis.

The quasi-static analysis assumed maximum energy transfer between the primary and secondary masses. The voltage, $V_{1 \mathrm{pl}}$, necessary to make the structure unstable throughout the entire gap was then calculated. A slower transition of the primary mass across the gap (non-ideal 
behavior) would act to decrease $\mathrm{V}_{1 \mathrm{PI}}$. This is because it would require a lower voltage to transfer less energy to the secondary mass.

There is some discrepancy in Fig. 9 between the measured and predicted AFR's below the transition frequency. The primary source of error is believed to be from the measurement of $\mathrm{x}_{1}$, the primary mass displacement. In measuring $\mathrm{Q}$, the primary and secondary mass displacements are measured when the structure is in the non-contact mode and operating near antiresonance. The primary mass motion at antiresonance is on the order of $1 \mu \mathrm{m}$, close to our test limit resolution of $0.25 \mu \mathrm{m}$. This could potentially introduce a significant measurement error in $\mathrm{x}_{1}$ and thus in Q. In Fig. 9, Eq. (29) is also plotted for $\mathrm{Q}=$ 15. This curve much more closely represents the measured AFR data.

Above the transition frequency, the measured data and the predicted AFR diverge because the drive voltage was not large enough to maintain the contact mode. A drive signal of 11 Volt was applied to the structure when the data was taken. As the frequency increased toward $f_{A R}$, the energy transfer between the primary and secondary masses increased. At the transition frequency, the secondary mass motion had become substantial enough such that the primary mass was unable to further compress the secondary spring and transfer more energy. Another way of expressing this is to say that a stable equilibrium point had been created in the gap because of an increase in the spring force $k_{2}$. The drive voltage was not large enough to maintain an unstable condition at every point in the gap. Increasing the drive voltage to maintain contact mode operation presented a difficulty. The primary mass frame that encircled the secondary mass limited the motion of the primary mass to $29 \mu \mathrm{m}$.

The extended life results, the power dissipation results, and the out-of-plane measurements indicate the potential this device has in gyroscope design. The device appears capable of sustained oscillations in the contact mode. The structure is also extremely robust to handling in the laboratory. A more formal approach to reliability is needed as a next step, and is left as future work. The power dissipation is low in the contact mode, presumably because of the native oxide layer that is present on polySi surfaces. The voltage pulse widths that are observed during the primary mass transition are very narrow in time, and there is no significant dc component of conduction at any reasonable operation frequency. There is, however, room for work in determining the source of conduction that is observed at very low frequencies. The small component of out-of-plane motion in the secondary mass is most likely limited by squeeze film damping. The secondary mass has very few etch release holes (spaced every $24 \mu \mathrm{m}$ ) that could account for the squeeze film layer.

\section{CONCLUSIONS}

Results were presented here of a device that is capable of large displacements in ambient atmospheric conditions. The focus of this work was on operating the SMD oscillator in the contact mode. The primary mass was allowed to contact the fixed drive electrodes, taking advantage of the nonlinear parallel-plate electrostatic forces. The analysis of the structure was presented showing the conditions necessary to maintain the contact mode. The system AFR was also derived and shows reasonable agreement with laboratory results. The system $Q$ was difficult to measure, owing to the fact that the primary mass motion was small near the antiresonant frequency. This structure has so far shown promise in the application of vibratory gyroscopes. The contact mode was low power, and the out-of-plane motion was minimal. The structure was also capable of extended operation.

\section{ACKNOWLEDGMENTS}

The authors would like to thank the entire staff of the Microelectronics Development Laboratory. Without their efforts in surface micromachining process development and fabrication, none of this work would have been possible. Sandia is a multiprogram laboratory operated by Sandia Corporation, a Lockheed Martin Company, for the United States Department of Energy under contract DE-AC0494AL85000.

\section{REFERENCES}

C. W. Dyck, J. J. Allen, and R. J. Huber, "Parallel-Plate Electrostatic Dual-Mass Oscillator," Proc. of the SPIE, vol. 3876, pp. 198-209.

R. S. Muller, "Microdynamics," Sensors and Actuators A, 21-23 (1990) pp. 1-8.

J. H. Smith, S. Montague, J. J. Sniegowski, J. R. Murray, and P. J. McWhorter, "Embedded Micromechanical Devices for Monolithic Integration of MEMS with CMO S," IEDM, 1995, pp. 25.3.1-25.3.4.

J. A. Geen, "A Path to Low Cost Gyroscopy," Solid-State Sensor and Actuator Workshop, June 1998, pp. 51-54.

E. S. Hung and S. D. Senturia, "Leveraged Bending for Full-Gap Positioning with Electrostatic Actuation, Solid-State Sensor and Actuator Workshop, June 1998, pp.83-86.

J. I. Seeger and B. E. Boser, "Dynamics and Control of Parallel-Plate Actuators Beyond the Electrostatic Instability," Transducers 99 Technical Digest, pp. 474-478.

H. C. Nathanson, W. E. Newell, R. A. Wickstrom, and J. R. Davis Jr., "The Resonant Gate Transistor," IEEE Transactions on Electron Devices, vol. ED-14, no. 3 (1967), pp. 117-133. 